\title{
Magnetic resonance spectroscopy of the frontal region in patients with metabolic syndrome: correlation with anthropometric measurement
}

\author{
Zizi Moustafa Hassan El-Mewafy ${ }^{1 B, C, F}$, Ahmed Abdel Khalek Abdel Razek ${ }^{2 A, B, C, D, E, F}$, \\ Mervat Mohamed El-Eshmawy ${ }^{10, E}$, Nader Ramadan Abo El-Eneen ${ }^{1 \mathrm{~A}, \mathrm{E}}$, Azaa Abdel Baky EL-Biaomy ${ }^{3, \mathrm{E}}$ \\ 'Department of Internal Medicine, Faculty of Medicine, Mansoura University, Egypt \\ 2Department of Diagnostic Radiology, Faculty of Medicine, Mansoura University, Egypt \\ ${ }^{3}$ Department of Clinical Pathology, Faculty of Medicine, Mansoura University, Egypt
}

\section{Abstract}

Purpose: To demonstrate ${ }^{1} \mathrm{H}-\mathrm{MR}$ spectroscopy of the frontal region in patients with metabolic syndrome and to correlate the metabolic ratios with anthropometric measurement.

\begin{abstract}
Material and methods: A prospective study was conducted upon 20 patients with metabolic syndrome (10 male, 10 female; mean age 52 years) and 20 age- and sex-matched volunteers. Patients were mild-moderate $(n=14)$ and marked and morbid obesity $(n=6)$. Patients and volunteers underwent ${ }^{1} \mathrm{H}-\mathrm{MR}$ spectroscopy of the frontal region. $\mathrm{The} \mathrm{Ch} / \mathrm{Cr}$ and $\mathrm{NAA} / \mathrm{Cr}$ ratio were calculated and correlated with anthropometric measurement.
\end{abstract}

Results: The Cho/Cr and NAA/Cr of patients with Mets (1.03 \pm 0.08 and $1.62 \pm 0.08)$ were significantly different ( $p=0.001)$ to those of volunteers $(0.78 \pm 0$ and $1.71 \pm 0.61$, respectively). The Cho/Cr and NAA/Cr cutoffs used to differentiate patients from volunteers were 0.89 and 1.77 with areas under the curve of 0.992 and 0.867 and accuracy of $97 \%$ and $93 \%$, respectively. There was a significant difference in $\mathrm{Cho} / \mathrm{Cr}$ and NAA/Cr between patients with marked-morbid obesity and moderate-mild obesity ( $p=0.001$ respectively).

Conclusions: We concluded that NAA/Cr and Cho/Cr ratios of the frontal region can differentiate patients with metabolic syndrome from volunteers and are well correlated with the anthropometric measurement.

Key words: MR spectroscopy, metabolic syndrome.

\section{Introduction}

Metabolic syndrome refers to the co-occurrence of several known cardiovascular risk factors, including insulin resistance, obesity, atherogenic dyslipidaemia, and high blood pressure. Metabolic syndrome may be important in the development of age-related cognitive decline, mild cognitive impairment, vascular dementia, and Alzheimer's disease [1-4]. Various imaging modalities are used to study the effect of metabolic syndrome on brain pa- renchyma. Magnetic resonance imaging studies including ${ }^{1} \mathrm{H}-\mathrm{MR}$ spectroscopy, magnetisation transfer ratios, voxel-based morphometry, and diffusion tensor imaging have been used to understand cerebral alterations of patients with metabolic syndrome [5-9]. The ${ }^{1} \mathrm{H}-\mathrm{MR}$ spectroscopy is diagnostic non-invasive imaging that offers the possibility of detecting and quantifying different metabolites of the brain [10-13]. Few studies are discussing the role of ${ }^{1} \mathrm{H}-\mathrm{MR}$ spectroscopy in assessment of metabolic changes of the brain in patients with metabolic syndrome [14-18].

Correspondence address:

Ahmed Abdel Khalek Abdel Razek, MD, Department of Diagnostic Radiology, Faculty of Medicine, Mansoura University, Mansoura, DK, Egypt 35512,

phone: 00201061948567, fax: 0020502315105, e-mail: arazek@mans.edu.eg

Authors' contribution:

A Study design · B Data collection · C Statistical analysis · D Data interpretation · E Manuscript preparation · F Literature search · G Funds collection 
The aim of this work is to demonstrate the role of ${ }^{1} \mathrm{H}-\mathrm{MR}$ spectroscopy of the frontal region in patients with metabolic syndrome and to correlate metabolic changes with anthropometric measurements.

\section{Material and methods}

\section{Patients}

Institutional board approval for the study and informed consent from each patient and volunteer was obtained. This prospective study was done on 23 adult patients with metabolic syndrome. The inclusion criteria were patients with metabolic syndrome diagnosed according to International Diabetes Federation [19]. We excluded three patients with diabetes mellitus. The final study group composed of 20 patients with Mets (10 male, 10 female; aged 45-60 years, mean 52 years), who underwent routine MR imaging and ${ }^{1} \mathrm{H}-\mathrm{MR}$ spectroscopy of the brain. Twenty age- and sex-matched healthy volunteers (10 male and 10 female; age range 45-60 years) were included as a control group. All patients and controls had excluded all diseases that can affect the central nervous system.

\section{Magnetic resonance imaging}

MR imaging was performed using a 1.5-tesla scanner and a commercially available circularly polarised head coil. MR imaging consisted of transverse T1- and T2-weighted imaging. The imaging parameters were TR/TE 500/14 ms for T1-weighted images and 5000/86 ms for T2-weighted images; field of view (FOV) $240 \times 240 \mathrm{~mm}$; section thickness $5 \mathrm{~mm}$ and intersection gap $0.5 \mathrm{~mm}$. The homogeneity of the magnetic field over the volume of interest was optimised by shimming. Suppression of the water signal was performed by using three preceding Gaussian pulses (60-Hz bandwidth). A single voxel point-resolved spectroscopy pulse sequence (PRESS) technique was applied using the following parameters: TR/TE 1500/35 ms, voxel size $8 \mathrm{~cm}^{3}$, and number of averages 128 . A voxel of $3 \times 3$ $\times 3 \mathrm{~cm}^{3}$ was located in the white matter of the left fron-

Table 1. The metabolic ratios at magnetic resonance spectroscopy of patients and volunteers

\begin{tabular}{|l|c|c|c|}
\hline Metabolites & Patients & Volunteers & $p$ value \\
\hline $\mathrm{Ch} / \mathrm{Cr}$ & $1.03 \pm 0.08$ & $0.78 \pm 0.05$ & 0.001 \\
\hline $\mathrm{NAA} / \mathrm{Cr}$ & $1.62 \pm 0.08$ & $1.71 \pm 0.61$ & 0.404 \\
\hline
\end{tabular}

Table 2. The receiver operating characteristic curve of metabolites in patients with Mets

\begin{tabular}{|l|c|c|c|c|c|}
\hline $\begin{array}{l}\text { Meta- } \\
\text { bolites }\end{array}$ & $\begin{array}{c}\text { Cutoff } \\
\text { point }\end{array}$ & AUC & Sensitivity & Specificity & Accuracy \\
\hline $\mathrm{Ch} / \mathrm{Cr}$ & 0.992 & 0.895 & $96.7 \%$ & $100.0 \%$ & $98.0 \%$ \\
\hline $\mathrm{NAA} / \mathrm{Cr}$ & 0.867 & 1.77 & $100.0 \%$ & $86.7 \%$ & $93.0 \%$ \\
\hline
\end{tabular}

tal region. A standard software package was used for post processing MR spectroscopic data.

\section{Image analysis}

Analysis of ${ }^{1} \mathrm{H}-\mathrm{MR}$ spectroscopy was performed by only one radiologist with 20 years of experience in MR imaging, and he was blinded to the clinical and laboratory findings of the patients. The images were transferred to a workstation (extended MR Workspace 2.6.3.5, Philips Medical Systems Nederland). Assignment of the resonance peaks of $\mathrm{N}$-acetyl-aspartate (NAA) at $2.02 \mathrm{ppm}$, creatine $(\mathrm{Cr})$ at $3.03 \mathrm{ppm}$ and $3.9 \mathrm{ppm}$, and choline $(\mathrm{Ch})$ at $3.2 \mathrm{ppm}$ were determined by fit and integration of the resonance in the post-processed Fourier spectra. The integrals of $\mathrm{Cho}$, $\mathrm{Cr}$ were calculated. The metabolite ratios of NAA/Cr and $\mathrm{Ch} / \mathrm{Cr}$ were calculated.

\section{Statistical analysis}

The statistical analysis of data was done by using Statistical Package for Social Science version 16.0 (SPSS Inc., Chicago, Ill, USA). The mean and standard deviation of $\mathrm{NAA} / \mathrm{Cr}$ and $\mathrm{Cho} / \mathrm{Cr}$ of patients and volunteers were calculated. The analysis of data was done to test the statistical significant difference. Independent sample (Student- $t$ test) and one-way ANOVA were done to study the difference of the metabolite ratios between patients and volunteers. The $\mathrm{p}$ value was considered significant when $\leq 0.05$. A receiver operating characteristic (ROC) curve was completed to evaluate the diagnostic capability of NAA/Cr and $\mathrm{Cho} / \mathrm{Cr}$ for differentiation of patients with metabolic syndrome from volunteers. The area under the curve, sensitivity, specificity, and accuracy were calculated. Pearson's correlation test was done to correlate NAA/Cr and Cho/Cr with anthropometric measurements. The correlation coefficient $r$ and $p$ value were calculated.

\section{Results}

Table 1 shows the metabolic ratios of $1 \mathrm{H}-\mathrm{MR}$ spectroscopy of patients and volunteers. Table 2 shows the receiver operating characteristic curve of metabolites ratio used to differentiate patients from volunteers. Figure 1 shows the ${ }^{1} \mathrm{H}-\mathrm{MR}$ spectroscopy of patients with metabolic syndrome and volunteers.

The mean $\mathrm{Cho} / \mathrm{Cr}$ ratio of the frontal region in patients with metabolic syndrome was $1.03 \pm 0.08$ and in volunteers was $0.78 \pm 0.05$. The cutoff $\mathrm{Cho} / \mathrm{Cr}$ used to differentiate patients with metabolic syndrome from volunteers was $\geq 0.895$ with an area under the curve of 0.992 , sensitivity of $100.0 \%$, specificity of $93.3 \%$, and accuracy of $97.0 \%$. The mean NAA/Cr ratio of the frontal region in patients with metabolic syndrome was $1.62 \pm 0.08$ and in volunteers was $1.71 \pm 0.61$. The cutoff NAA/Cr used to differentiate patients with metabolic syndrome from vol- 


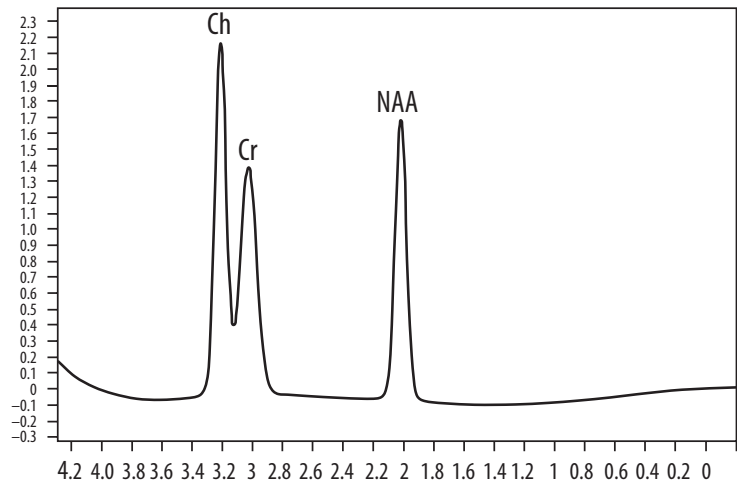

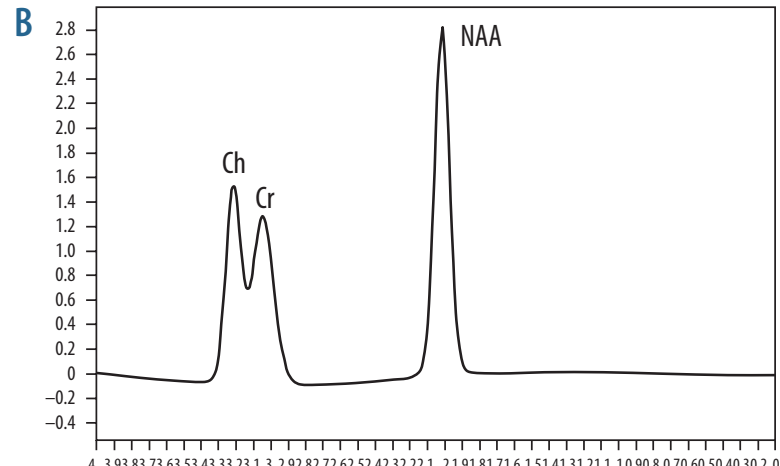

Figure 1. Magnetic resonance (MR) spectrum of frontal region. A) MR spectroscopy of the frontal region in patients with metabolic syndrome $(\mathrm{NAA} / \mathrm{Cr}=1.63, \mathrm{Ch} / \mathrm{Cr}=1.07)$. B) MR spectroscopy of the frontal region in healthy volunteers $(\mathrm{NAA} / \mathrm{Cr}=1.71, \mathrm{Ch} / \mathrm{Cr}=0.78)$
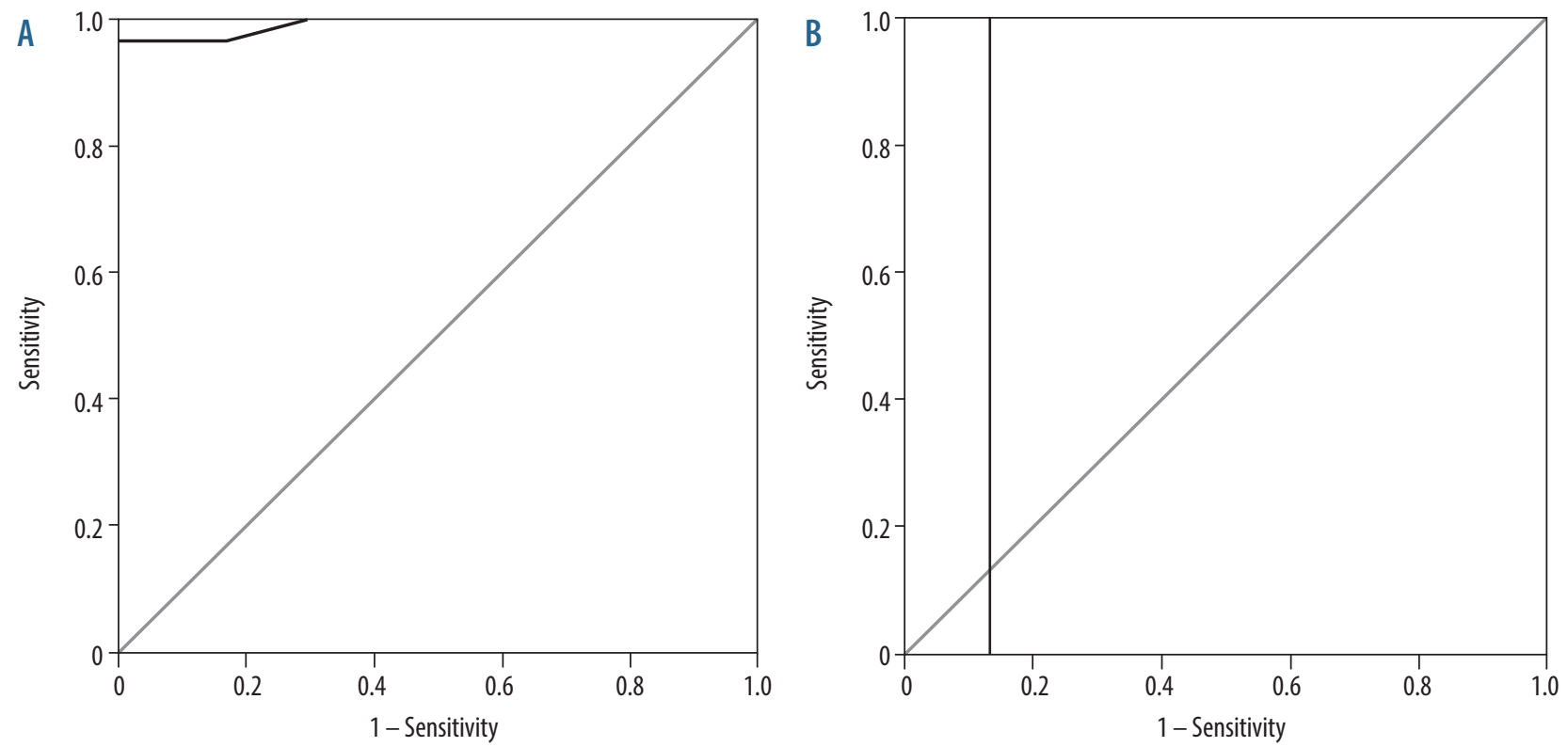

Figure 2. Receiver operating characteristic (ROC) curve of patients versus volunteers. A) The cutoff Cho/ $\mathrm{Cr}$ of the frontal region used to differentiate patients with metabolic syndrome from volunteers was $\geq 0.895$ with an area under the curve of 0.992 , sensitivity of $100.0 \%$, specificity of $93.3 \%$, and accuracy of $97.0 \%$. B) The cutoff NAA/Cr of the frontal lobe used to differentiate patients with metabolic syndrome from volunteers was $\leq 1.77$ with an area under the curve of 0.867 , sensitivity of $100.0 \%$, specificity of $86.7 \%$, and accuracy of $93.0 \%$

unteers was $\leq 1.77$ with an area under the curve of 0.867 , sensitivity of $100.0 \%$, specificity of $86.7 \%$, and accuracy of 93.0\% (Figure 2, Table 2).

Patients were mild-moderate $(n=14)$ and marked and morbid obesity $(n=6)$. The mean Cho/Cr of the frontal region was $1.09 \pm 0.09$ and $0.98 \pm 0.03$ in severe and mild obesity, respectively. The mean NAA/Cr ratio in the frontal region was $1.56 \pm 0.07$ and $1.67 \pm 0.03$ in severe and mild obesity, respectively (Table 3 ). There was a statistically significant difference in brain metabolite ratios between patients with severe obesity and patients with mild obesity $(p=0.001)$.

\section{Discussion}

The main findings in this study is that there is a significant difference in the brain metabolite ratios of patients with metabolic syndrome compared to volunteers, and patients with severe obesity compared to mild obesity. There is a correlation of brain metabolite ratios with anthropometric measures in patients with metabolic syndrome.

In this study, the mean metabolite ratios of the frontal region in patients with metabolic syndrome were different than that of volunteers. This may be attributed to visceral obesity, insulin resistance, atherogenic dyslipidaemia, and endothelial dysfunction, as well as a constellation of independent factors (e.g. molecules of hepatic, vascular, and immunological origin) that mediate specific components of the metabolic syndrome. A number of theories have

Table 3. The metabolic ratios at magnetic resonance spectroscopy of patients with mild and morbid obesity

\begin{tabular}{|l|c|c|c|}
\hline Metabolites & Mild obesity & Morbid obesity & $p$ value \\
\hline $\mathrm{Ch} / \mathrm{Cr}$ & $0.98 \pm 0.03$ & $1.09 \pm 0.09$ & 0.001 \\
\hline $\mathrm{NAA} / \mathrm{Cr}$ & $1.67 \pm 0.03$ & $1.56 \pm 0.07$ & 0.001 \\
\hline
\end{tabular}


been proposed for the ill effects of metabolic syndrome on the brain and cognition, including neuro-inflammation, oxidative stress, abnormal brain lipid metabolism, and impaired vascular reactivity [9].

In this study, there was decreased NAA/Ch ratio of the frontal region in patients compared to volunteers. The NAA is an amino acid that is exclusively synthesised and localised in neurons. It acts as a major osmolyte and serves as a marker of neuronal viability. The primary role of NAA is neuronal-glial cell specific signalling for optimal functioning of the central nervous system. The decline in brain NAA reflects axonal or neuronal dysfunction or loss in neuronal density $[10,11]$. Lower NAA is consistent with derangement of neurometabolism, lower dendritic/axonal density, and/or axonal loss. Decreased NAA might be indicative of decreased neuronal viability in metabolic syndrome as a result of chronic hypoxia and hyperglycaemia [14-18].

In this study, the Cho/Cr ratio was higher in patients than in volunteers. Previous studies reported that choline-containing compounds are an indicator of cell density and cell wall turnover. They also reflect metabolism of myelin and other phospholipids of the cell membrane [10-14]. The high choline level of the frontal white matter suggests membrane and/or myelin alterations and/or alterations in membrane turnover. Thus, these results point to axonal and myelin abnormalities of the frontal white matter, as well as decreased neuronal viability in the frontal lobe [14-17].

In this study, there was a decrease in NAA/Cr levels and an increase in the Cho/Cr ratio in patients with se- vere morbid obesity compared to mild-moderate obesity. This is attributed to the fact that obesity contributes to hypertension, high serum cholesterol, low HDL cholester$\mathrm{ol}$, and hyperglycaemia. Visceral obesity causes a decrease in insulin-mediated glucose uptake and is clearly related to insulin resistance. The mechanisms for this probably involve adipokines (made by adipose tissue), which modulate crosstalk between metabolism and vascular function [1-5].

This study has some limitations. First, the small number of patients limits the ability to achieve statistically significant results. However, further multi-centre studies upon a larger number of patients with metabolic syndrome are recommended. Second, this study applied single-voxel proton MR spectroscopy of the brain. Further studies with application of multi-parametric imaging of the brain combined with diffusion-weighted MR imaging [20-22], diffusion tensor MR imaging [23,24], and dynamic contrast MR imaging [25-27] with a higher three-tesla machine [28-31] may provide better results.

\section{Conclusions}

We conclude that the NAA/Cr and Cho/Cr ratios of the frontal region can differentiate patients with metabolic syndrome from healthy volunteers and are well correlated with anthropometric measures.

\section{Conflict of interest}

The authors report no conflict of interest.

\section{References}

1. Samson SL, Garber AJ. Metabolic syndrome. Endocrinol Metab Clin North Am 2014; 43: 1-23.

2. Eckel RH, Grundy SM, Zimmet PZ. The metabolic syndrome. Lancet 2005; 365: 1415-1428

3. Oda E. Metabolic syndrome: its history, mechanisms, and limitations. Acta Diabet 2012; 49: 89-95.

4. Moran C, Beare R, Phan TG, et al. Type 2 diabetes mellitus and biomarkers of neurodegeneration. Neurology 2015; 85: 1123-1130.

5. Yu Y, Sun Q, Yan LF, et al. Multimodal MRI for early diabetic mild cognitive impairment: study protocol of a prospective diagnostic trial. BMC Med Imaging 2016; 16: 50.

6. Tan X, Fang P, An J, et al. Micro-structural white matter abnormalities in type 2 diabetic patients: a DTI study using TBSS analysis. Neuroradiology 2016; 58: 1209-1216.

7. deBresser J, Reijmer YD, van den Berg E, et al. Microvascular determinants of cognitive decline and brain volume change in elderly patients with type 2 diabetes. Dement Geriatr Cogn Disord 2010; 30: 381-386.

8. Sims RC, Katzel LI, Lefkowitz DM, et al. Association of fasting glucose with subclinical cerebrovascular disease in older adults without Type 2 diabetes. Diabet Med 2014; 31: 691-698.
9. Chen Y, Liu Z, Zhang J, et al. Selectively Disrupted Functional Connectivity Networks in Type 2 Diabetes Mellitus. Front Aging Neurosci 2015; 7: 233.

10. Razek AA, Poptani H. MR spectroscopy of head and neck cancer. Eur J Radiol 2013; 82: 982-989.

11. Razek AA, Nada N. Correlation of Choline/Creatine and Apparent Diffusion Coefficient values with the prognostic parameters of Head and Neck Squamous Cell Carcinoma. NMR Biomed 2016; 29: 483-489.

12. Abdel Razek A, Abdalla A, Abdel Gaber N, et al. Proton MR Spectroscopy of the brain in children with neuronopathic Gaucher's disease. Eur Radiol 2013; 23: 3005-3011.

13. Razek AA, Abdalla A, Ezzat A, et al. Minimal hepatic encephalopathy in children with liver cirrhosis: diffusion-weighted MR imaging and proton MR spectroscopy of the brain. Neuroradiology 2014; 56: 885-891.

14. Sinha S, Ekka M, Sharma U, et al. Assessment of changes in brain metabolites in Indian patients with type-2 diabetes mellitus using proton magnetic resonance spectroscopy. BMC Res Notes 2014; 7: 41.

15. Tiehuis A, van der Meer F, Mali W, et al. MR spectroscopy of cerebral white matter in type 2 diabetes; no association with clinical variables and cognitive performance. Neuroradiology 2010; 52: 155-1561. 
16. Modi S, Bhattacharya M, Sekhri T, et al. Assessment of the metabolic profile in Type 2 diabetes mellitus and hypothyroidism through proton MR spectroscopy. Magn Reson Imaging 2008; 26: 420-425.

17. Sahin I, Alkan A, Keskin L, et al. Evaluation of in vivo cerebral metabolism on proton magnetic resonance spectroscopy in patients with impaired glucose tolerance and type 2 diabetes mellitus. J Diabet Comp 2008; 22: 254-260.

18. Ajilore O, Haroon E, Kumaran S, et al. Measurement of brain metabolites in patients with type 2 diabetes and major depression using proton magnetic resonance spectroscopy. Neuropsychopharmacology 2007; 32: 1224-1231.

19. http://www.idf.org/webdata/docs/Metac_syndrome_def.pdf. Accessed: 23 October 2013

20. Abdel Razek AA, Elkammary S, Elmorsy AS, et al. Characterization of mediastinal lymphadenopathy with diffusion-weighted imaging. Magn Reson Imaging 2011; 29: 167-172.

21. Abdel Razek A, Samir S, El-Said A. Role of diffusion-weighted MR imaging in differentiation of Graves' disease from painless thyroiditis. Polish J Radiol 2017; 30: 230-234.

22. Abdel Razek A, Mazroa J, Baz H. Assessment of white matter integrity of autistic preschool children with diffusion weighted MR imaging. Brain Dev 2014; 36: 28-34.

23. Abdel Razek A, Al-Adlany M, Alhadidy A, et al. Diffusion tensor imaging of the renal cortex in diabetic patients: correlation with urinary and serum biomarkers. Abdom Radiol 2017; 42: 1493-1500.
24. El-Serougy L, Abdel Razek AA, Ezzat A, et al. Assessment of diffusion tensor imaging metrics in differentiating low-grade from highgrade gliomas. Neuroradiol J 2016; 29: 400-407.

25. Abdel Razek AA, Mousa A, Farouk A, et al. Assessment of semiquantitative parameters of dynamic contrast-Enhanced Perfusion MR Imaging in Differentiation of Subtypes of Renal Cell Carcinoma. Polish J Radiol 2016; 81: 90-94.

26. Abdel Razek AA, Gaballa G. Role of perfusion magnetic resonance imaging in cervical lymphadenopathy. J Comput Assist Tomogr 2011; 35: 21-25.

27. Razek AA, Elsorogy LG, Soliman NY, et al. Dynamic susceptibility contrast perfusion MR imaging in distinguishing malignant from benign head and neck tumors: a pilot study. Eur J Radiol 2011; 77: 73-79.

28. Abdel Razek AA, Elkhamary S, Al-Mesfer S, et al. Correlation of apparent diffusion coefficient at $3 \mathrm{~T}$ with prognostic parameters of retinoblastoma. AJNR Am J Neuroradiol 2012; 33: 944-948.

29. Abdel Razek AA, Gaballa G, Denewer A, et al. Diffusion weighted MR imaging of the breast. Acad Radiol 2010; 17: 382-386.

30. Razek AA, Sieza S, Maha B. Assessment of nasal and paranasal sinus masses by diffusion-weighted MR imaging. J Neuroradiol 2009; 36: 206-211.

31. Razek AA. Diffusion magnetic resonance imaging of chest tumors. Cancer Imaging 2012; 12: 452-463. 